\title{
PERCEPCIONES DE LOS DOCENTES EN PRE- SERVICIO RELATIVAS A LA INCLUSIÓN Y LA ATENCIÓN A LA DIVERSIDAD
}

\author{
Carla Leticia Paz \\ Universidad Pedagógica Nacional Francisco Morazán \\ cpaz@upnfm.edu.hn \\ María Cristina Cardona Moltó \\ Universidad de Alicante \\ cristina.cardona@ua.es
}

\section{RESUMEN}

El reconocimiento de la diversidad humana como valor educativo, ha implicado que los docentes de las aulas regulares, reciban preparación para crear espacios de aprendizaje inclusivos. Siguiendo estas tendencias, la Universidad Pedagógica Nacional Francisco Morazán, incluyó en su reciente reforma a los planes de estudio, competencias docentes vinculadas con la atención a la diversidad y la inclusión. En esta investigación se analizaron las percepciones de los docentes en pre servicio de la UPNFM, relacionadas con los principios teóricos y metodológicos de la atención a la diversidad, la formación recibida en la Universidad en estos ámbitos y finalmente las competencias que valoran como importantes para desarrollar la educación inclusiva. Además de ello se realizó una comparación de estas percepciones por ámbito de estudio y pertenencia a grupo étnico. El estudio es de naturaleza cuantitativa, no experimental y de corte transversal, participaron 470 estudiantes en práctica profesional II.

\section{PALABRAS CLAVE}

Inclusión, Atención a la Diversidad, Percepciones, Formación Inicial, Docentes en Pre-Servicio

\begin{abstract}
Recognition of human diversity and educational value, has meant that teachers in regular classrooms will receive preparation for creating inclusive learning spaces. Following these trends, the National Pedagogical University Francisco Morazán included in its recent reform to the study programs, teaching skills linked to attention to diversity and inclusion.
\end{abstract}


Recibido 21 de septiembre de 2016 /Aceptado 9 de noviembre de2016

In this research, the perceptions of UPNFM pre service teachers related to the theoretical and methodological principles of the Attention to Diversity were analyzed, the training received at the University in these areas, and finally, the competences that are considered important to develop an inclusive education. In addition, a comparison of these perceptions by field of study and membership of an ethnic group was held. The study is quantitative, not experimental and cross-sectional, 470 students from the Professional Practicum II participated.

\section{KEYWORDS}

Inclusion, Attention to Diversity, Perceptions, Initial Training Pre-Service Teachers

\section{INTRODUCCIÓN}

Uno de los grandes desafíos hoy en día para las instituciones de formación docente, radica en la preparación inicial del profesorado para la atención a la diversidad del alumnado. El reconocimiento de los docentes como factor clave en el desarrollo de la inclusión educativa, es uno de los importantes avances en los últimos años. Después de una época en que las prioridades de las reformas educativas en América Latina y el Caribe estuvieron centradas en infraestructura, textos, equipos, laboratorios, y otros insumos (Campos, 2003), la región se encuentra en una etapa en la que se realizan esfuerzos por mejorar la equidad, calidad y eficacia de los aprendizajes en un marco de justicia social.

Este esfuerzo se pone de manifiesto en las Declaraciones de Jomtiem (1990) y Dakar (2000) en el caso de América; en el Proyecto Regional de Educación para América Latina y el Caribe, PRELAC (La Habana, 2002), así como en los múltiples foros internacionales en los que se ha analizado y reflexionado el protagonismo de los docentes en las transformaciones educativas que las sociedades y las comunidades demandan (UNESCO, 2012). Entre ellas hay que destacar la acogida y la atención educativa de calidad que requiere un alumnado caracterizado por su diversidad individual y colectiva. 
Desde la mirada de la educación inclusiva, la diversidad del alumnado se considera un elemento enriquecedor del proceso enseñanza. Será entonces indispensable que el profesorado de educación regular, perciba como provechosa la atención a las diferencias humanas, lo contrario significaría la reproducción de prácticas educativas que en el pasado han generado discriminación y exclusión.

Reorientar la formación del profesorado, hacia el desarrollo de creencias y percepciones favorables con la inclusión educativa, el reconocimiento y aprecio por la diversidad, es fundamental ya que estas, están vinculadas con las expectativas que se tienen de los estudiantes, (Mason, 1997; Wiggins y Follo, 1999). Por otro lado, si la diversidad está en las escuelas, la formación para responder a ella no debe dirigirse sólo a los especialistas, sino que es esencial también para el profesorado del aula regular ( León, 1998). En última instancia los profesores no pueden enseñar lo que desconocen. Si las universidades e instituciones de formación cambian su currículum de forma y manera que se haga eco de la diversidad del alumnado al que sirven, estarán cumpliendo con su misión (Nieto y Santos, 1997).

En correspondencia con estas tendencias internacionales, desde el año 2002, la Universidad Pedagógica Nacional Francisco Morazán (UPNFM), a través de la Carrera de Educación Especial crea el Proyecto Atención a la Diversidad, cuyo propósito fundamental es la atención a los ámbitos de diversidad humana presente en la población estudiantil de la institución, y a su vez se espera contribuir con la formación inicial en materia de inclusión y atención a la diversidad (Documento Base Proyecto Atención a la Diversidad, 2002). Sumado a ello, de manera reciente la UPNFM desarrolló un proceso de rediseño de los planes de estudio, incorporando una competencia genérica, relacionada con el respeto y valoración de la diversidad, la cual se conceptualiza como la capacidad de comprender y aceptar la diversidad física, psicológica, social y cultural como un componente enriquecedor personal y colectivo con el fin de promover la convivencia en paz entre las personas sin incurrir en distinciones de género, edad, religión, etnia, condición social y política (Aguilar, 2008). Sin embargo, hasta la fecha no se ha valorado sí estos esfuerzos han contribuido a desarrollar percepciones y conocimientos favorables y necesarios para la 
atención a la diversidad en contextos inclusivos. Este precisamente es el motivo que ha impulsado el desarrollo de esta investigación que se ha guiado por los siguientes objetivos:

- Identificar las percepciones de los docentes en pre-servicio de la UPNFM, relativas a los principios teóricos y metodológicos de la atención a la diversidad y la inclusión.

- Determinar las percepciones de los docentes en pre-servicio sobre los conocimientos adquiridos durante la formación en la UPNFM en materia de inclusión y atención a la diversidad.

- Identificar las percepciones de los docentes en pre-servicio sobre las competencias específicas necesarias para atender la diversidad en ambientes inclusivos.

- Comparar por ámbito estudio y pertenencia a un grupo étnico, las percepciones de los docentes en pre-servicio, sobre la atención a la diversidad y la inclusión.

\section{MÉTODOS Y MATERIALES}

\subsection{Diseño}

Se optó por un diseño no experimental descriptivo- comparativo mediante encuesta. Los diseños no experimentales describen alguna circunstancia que ha ocurrido o bien examina las relaciones entre aspectos sin ninguna manipulación directa de las condiciones que son experimentadas (Cardona, 2002). El diseño de encuesta fue de corte transversal pues la medición se efectuó en un único momento, sin aplicaciones posteriores. La comparación de las percepciones de los estudiantes acerca de los principios de la atención a la diversidad y la inclusión se realizó en función de dos variables independientes; (1) el área de conocimientos (Ciencias Sociales y Educación, Educación Física, Arte e idiomas, y Ciencia y Tecnología), (2) el grupo étnico (mayoritario y minoritario). Este diseño permite establecer comparaciones entre los grupos en estudio. 


\subsection{Población}

Como sujetos participantes, se seleccionó la población total de estudiantes de último año de carrera de la Sede Central de la UPNFM. Debido a que este grupo de estudiantes había cursado el total de las asignaturas que componen el plan de estudios, se convirtieron en los informantes claves para esta investigación. En el año 2012, el centro contaba con una población de $N=545$ estudiantes de último año.

De esta población participaron 470 estudiantes de cuarto año de las diferentes carreras de la UPNFM, lo que representa el $86 \%$ de la población. Hay que mencionar que en el momento de realizar el estudio algunos participantes se encontraban realizando unos seminarios prácticos en diversos centros educativos, por lo que no todos pudieron acudir al llamamiento.

\subsection{Entorno}

Esta investigación se realizó en la sede central, modalidad presencial de la Universidad Pedagógica Nacional Francisco Morazán de Honduras (UPNFM), institución que constituye el único centro de educación universitaria centroamericano dedicado exclusivamente a la formación de docentes para todos los niveles de la enseñanza

\subsection{Experimentos}

Para realizar el estudio de las percepciones de los docentes en pre servicio sobre la educación inclusiva y atención a la diversidad, se identificó, el cuestionario como instrumento más apropiado ya que permite llegar a un mayor número de sujetos y ofrece información detallada sobre los participantes en un tiempo relativamente corto. En tal sentido, se realizó una revisión previa de la literatura con la intención de identificar un instrumento que nos permitiera medir la variable en cuestión. Aunque se identificaron algunos instrumentos, ninguno de ellos cuenta con aspectos relacionados a la formación recibida sobre la inclusión y la atención a la diversidad, A ello hay que agregar que el presente estudio se realiza con la finalidad de evaluar el proceso formativo que se brinda, en particular, a los estudiantes de la UPNFM. De ahí, que se estimará conveniente la 
elaboración de una escala diseñada ad hoc para medir las variables de interés contenidas en los objetivos de investigación.

La Escala de Percepciones acerca de la Inclusión y la Atención a la Diversidad (PIAD, Paz y Cardona, 2014)) consta 23 ítems y está estructurada en 3 sub-escalas, utilizando una escala tipo Likert de 4 puntos ( 1 = totalmente en desacuerdo; $2=$ en desacuerdo; $3=$ de acuerdo; 4 = mиy de acuerdo). La administración de la escala era sencilla y el tiempo para completarla era aproximadamente de 20 minutos. P ara estudiar la Validez de Contenido de la escala se sometió la misma a una evaluación por jueces expertos. El índice de validez de contenido por las tres partes de la prueba fue de, 0.88, 0.77, 0.80, respectivamente y el índice general fue de 0.81 , lo que hace que la escala PIAD se considere aceptable y valida a nivel de contenido. Se desarrolló una prueba piloto para determinarla confiabilidad, obteniendo un alpha de Cronbach de 0.85, índice que se considera aceptable cuando la variable que se mide son percepciones y el número de ítems en el instrumento es reducido (Kerlinger y Lee, 2002).

\subsection{Análisis estadístico}

Se realizó un análisis cuantitativo a través del paquete estadístico IBM, SPSSStatistics 21 (StatisticalPackagefor Social Science). El procedimiento de análisis incluyó, descriptivos como: cálculo de frecuencias y porcentajes, cálculo de medidas de tendencia central, en este caso se hizo uso de la media aritmética y en el cálculo de medidas de dispersión de la desviación típica y para comparar las percepciones de los estudiantes por área de conocimientos y grupo étnico, se emplearon pruebas estadísticas paramétricas, específicamente, el análisis de la varianza de dos factores.

\section{DISCUSIÓN TEÓRICA}

El reconocimiento del valor de la diversidad humana en educación y la creación de escuelas inclusivas genera nuevas necesidades de formación docente. Para Sales (2006), estas necesidades consisten en: (1) el desarrollo de programas de formación y actualización que hagan posible la redefinición del papel del profesorado y permitan afrontar sus nuevas 
funciones para la atención a la diversidad; (2) la promoción de actitudes positivas ante la diversidad y la comprensión profunda de los cambios organizativos y curriculares que exige una educación inclusiva; (3) el desarrollo de una formación básica para todos los profesionales de la educación que evite las perspectivas categoriales centradas en el déficit, el aislamiento y la descoordinación entre profesionales (generalistas y especialistas).

Actualmente se considera que todo profesor debe tener una formación básica para trabajar en contextos de diversidad. De esta manera, frente a visiones categoriales y homogeneizantes, las más recientes tendencias formativas plantean una visión integral más cercana a la formación centrada en la escuela, la investigación-acción, y el análisis institucional o desarrollo organizativo (Sales, 2006; Torres, 2009). Esta formación inicial debe ser polivalente, no categórica y, por tanto, centrada en las necesidades de los estudiantes y no en su condición deficitaria (Arranz, 2003).

En este sentido, la educación inclusiva conlleva un nuevo rol docente. Se considera que el profesor del aula regular es el elemento clave del proceso de atención a la diversidad. Tal como sugiere Parrilla (2003), es necesario forjar una nueva identidad docente: competente pedagógicamente, capaz de investigar y reflexionar sobre la práctica con otros profesores y consciente de las facetas sociales y morales de su profesión. De este planteamiento, se derivan algunos elementos esenciales para la formación inicial del profesorado, propuestos por Durán y Giné (2011):

- Aceptación de todo el alumnado como propio. Los alumnos y alumnas del grupo clase son responsabilidad del profesor, independientemente de las características personales que tengan. En algunos casos, la atención educativa puede ser compartida con otros profesores de apoyo, pero ello no debe significar la derivación ni el desentendimiento del profesor de aula ordinaria.

- Aula y centro ordinario como espacio preferente de atención. Los alumnos deben hallar la atención a sus necesidades educativas en entornos lo más normalizados posibles, con los apoyos necesarios. La escolarización en centros de educación especial debe 
reservarse exclusivamente para aquellos alumnos para los cuales los centros ordinarios hayan agotado toda su capacidad de atención.

- Conocimiento sobre las diferencias de los alumnos. El profesorado debe conocer a sus estudiantes en términos de potencialidades y necesidades.

- Estrategias para la inclusión. Para facilitar la atención a la diversidad es necesario el dominio de decisiones curriculares y metodologías que faciliten el mayor grado de participación y aprendizaje de todos.

\section{RESULTADOS}

El primer objetivo de esta investigación, pretendía identificar la percepción de los estudiantes acerca de los planteamientos teóricos y metodológicos propios de la educación inclusiva y de la atención a la diversidad del alumnado. Como se observa en la Tabla 1, los docentes en pre-servicio tienen una percepción global claramente favorable de los principios que orientan la atención a la diversidad en contextos inclusivos $(M=32.00 ; D T$ =4.26), siendo la puntuación mínima de 9 y la máxima de 36 .

Tabla 1. Percepciones sobre los planteamientos teórico metodológicos de la inclusión y atención a la diversidad

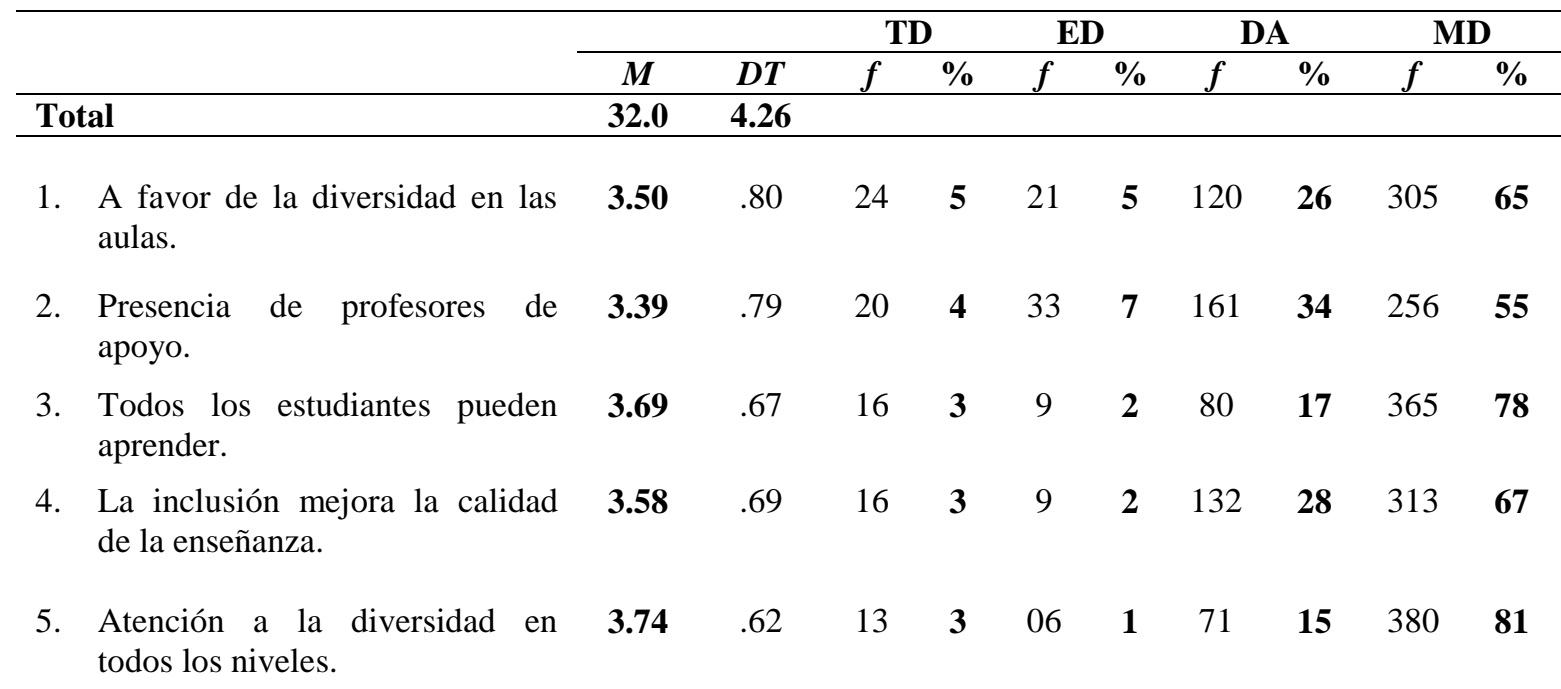


6. Todos los profesores en formación deben recibir contenidos sobre educación especial.

7. Planes de estudio de profesorado que contengan temas relacionados con la atención a la diversidad.

8. Aceptaría compartir la docencia con un profesor de apoyo.

9. La inclusión y la atención a la diversidad deberían ser ejes transversales en los planes de estudio.

3.73

.63

12

3

12

$3 \quad 67$

14379

81

3.53

.74

19

414

3

138

29299

64

$\begin{array}{llllllllll}\mathbf{3 . 1 6} & .87 & 28 & \mathbf{6} & 63 & \mathbf{1 3} & 187 & \mathbf{4 0} & 192 & \mathbf{4 1} \\ \mathbf{3 . 4 7} & .66 & 8 & \mathbf{5} & 21 & \mathbf{5} & 185 & \mathbf{2 6} & 256 & \mathbf{6 5}\end{array}$

$\mathrm{TD}=$ Totalmente en desacuerdo $; E D=$ En desacuerdo $; \mathrm{DA}=$ De acuerdo $; \mathrm{MA}=$ Muy de acuerdo

Todos los ítems que medían este factor fueron valorados con puntajes por encima del punto medio de la escala. Sin embargo, aunque las percepciones acerca de los principios de la inclusión parecen positivas, sólo un $41 \%$ de los respondientes estaban muy de acuerdo en compartir la docencia con un profesor de apoyo.

Otro de los propósitos de este estudio fue analizar en qué medida los estudiantes habían recibido contenidos formativos relacionados con la puesta en práctica de la educación inclusiva y la atención a la diversidad. La puntuación total obtenida de la suma de todos los ítems de esta subescala $(M=14.50 ; D T .=3.73)$ resultó ser levemente inferior al punto medio situado en 15 (mín. 6 - máx. 24). Esto indica que los estudiantes tenían un conocimiento bajo del uso de estrategias para implementar la educación inclusiva y atender la diversidad. Como puede visualizarse en la Tabla 2, en ninguna de las estrategias consideradas básicas para implementar la inclusión se había recibido formación suficiente, a juicio de los respondientes

Tabla 2. Contenidos formativos recibidos sobre inclusión y atención a la diversidad

\begin{tabular}{llllllllll} 
& \multicolumn{2}{c}{ NA } & \multicolumn{2}{c}{ AL } & \multicolumn{2}{c}{ BA } & \multicolumn{2}{c}{ MU } \\
\hline$M$ & $D T$ & $f$ & $\%$ & $f$ & $\%$ & $f$ & $\%$ & $f$ & $\%$ \\
\hline
\end{tabular}

Total

$14.5 \quad 3.73$

1.Diseño de adaptaciones curriculares

$2.15 \quad .88$

113

24

213

45

$\begin{array}{lll}04 & 22 & 40\end{array}$

09 


\begin{tabular}{lllllllllll}
$\begin{array}{l}\text { 2.Implementación de adaptaciones } \\
\text { instructivas }\end{array}$ & $\mathbf{2 . 5 0}$ & .88 & 58 & $\mathbf{1 2}$ & 185 & $\mathbf{3 9}$ & 161 & $\mathbf{3 4}$ & 66 & $\mathbf{1 4}$ \\
$\begin{array}{l}\text { 3.Estrategias organizativas } \\
\text { (agrupamiento) }\end{array}$ & $\mathbf{2 . 5 9}$ & .89 & 50 & $\mathbf{1 1}$ & 171 & $\mathbf{3 6}$ & 170 & $\mathbf{3 6}$ & 79 & $\mathbf{1 7}$ \\
$\begin{array}{l}\text { 4.Estrategias de enseñanza } \\
\text { colaborativa }\end{array}$ & $\mathbf{2 . 5 5}$ & .85 & 50 & $\mathbf{1 1}$ & 173 & $\mathbf{3 7}$ & 185 & $\mathbf{3 9}$ & 62 & $\mathbf{1 3}$ \\
$\begin{array}{l}\text { 5. Estrategias y recursos de evaluación } \\
\text { auténtica. }\end{array}$ & $\mathbf{2 . 6 9}$ & .88 & 43 & $\mathbf{0 9}$ & 149 & $\mathbf{3 2}$ & 188 & $\mathbf{4 0}$ & 90 & $\mathbf{1 9}$ \\
$\begin{array}{l}\text { 6. Procesos de reflexión crítica sobre } \\
\text { estereotipos y creencias acerca de la } \\
\text { diversidad. }\end{array}$ & $\mathbf{2 . 1 1}$ & .90 & 127 & $\mathbf{2 7}$ & 204 & $\mathbf{4 3}$ & 98 & $\mathbf{2 1}$ & 41 & $\mathbf{0 9}$ \\
\hline
\end{tabular}

$1=$ Nada $; 2=$ Algo $; 3=$ Bastante $; 4$ = Mucho

El tercer objetivo pretendía identificar aquellas competencias que los estudiantes consideran necesarias para atender la diversidad en contextos inclusivos. Todas las competencias fueron consideradas importantes, sin embargo, las mejor valoradas para el desempeño docente fueron, en primer lugar, ítem 8 la 'Capacidad para reflexionar críticamente sobre las actitudes y valores relacionados con la diversidad' $(M=8.57 ; D T=$ 1.51); en segundo lugar, ítem 2 la 'Capacidad para diseñar y aplicar estrategias de atención en contextos culturales y sociales diversos' ( $M=8.48 ; D T=1.62) \mathrm{y}$; en tercer lugar, ítem 4 la 'Capacidad para realizar adaptaciones instructivas en el aula de clase' $(\mathrm{M}=8.47 ; \quad D T=$ 1.56). Los resultados se presentan en la Tabla 3.

Tabla 3. Competencias necesarias para la atención a la diversidad

\begin{tabular}{|c|c|c|c|c|}
\hline \multirow[b]{2}{*}{ Total } & $M$ & $D T$ & Min & $\operatorname{Max}$ \\
\hline & 66.9 & 10.2 & & \\
\hline 1. Capacidad para valorar la diversidad del alumnado. & & & & \\
\hline & 8.27 & 1.56 & 2 & 10 \\
\hline $\begin{array}{l}\text { 2. Capacidad para diseñar y aplicar estrategias de atención en contextos } \\
\text { culturales y sociales diversos. }\end{array}$ & 8.48 & 1.62 & 1 & 10 \\
\hline 3. Capacidad para diseñar e implementar adaptaciones curriculares. & 8.23 & 1.79 & 1 & 10 \\
\hline 4. Capacidad para realizar adaptaciones instructivas en el aula de clase. & 8.47 & 1.66 & 1 & 10 \\
\hline 5. Capacidad para implementar estrategias organizativas. & 8.26 & 1.70 & 1 & 10 \\
\hline 6. Capacidad para desarrollar procesos colaborativos con otros profesores. & 8.17 & 1.76 & 1 & 10 \\
\hline 7. Capacidad para implementar estrategias y recursos de evaluación auténtica. & 8.32 & 1.84 & 2 & 10 \\
\hline
\end{tabular}


8. Capacidad para reflexionar críticamente sobre las actitudes, valores y acciones relacionadas con la atención a la diversidad.

En cuanto al cuarto objetivo que pretendía la comparación de las percepciones por ámbito de estudio y pertenencia a un grupo étnico, se determinó que ni el efecto principal de la variable ámbito de estudio $[F(2,469)=.56, p=.570]$, ni el efecto principal de la variable grupo étnico $[F(1,469)=.01, p=.924]$ resultaron estadísticamente significativos. Estos resultados sugieren que las percepciones acerca de los principios de la inclusión que tienen los estudiantes, no diferían significativamente. Igualmente, el efecto de la interacción A x G no alcanzó la significación estadística $[F(2,469)=.61, p=.544]$ ni sobre el factor en su conjunto ni atendiendo a sus ítems por separado, exceptuado el ítem 8 'Aceptaría compartir la docencia con un profesor de apoyo'. El análisis post hoc de las medias de este ítem empleando el test de Scheffé indicaron que la del grupo de participantes que estudiaba Educación Física, Arte e Idiomas del grupo mayoritario $(M=3.22 ; D T=.87)$ fue significativamente diferente de la del grupo minoritario $(M=2.95 ; D T=.81)$, mientras que en los otros dos grupos CC. SS. y Educación y Ciencia y Tecnología no se apreciaron diferencias con significación estadística entre los grupos mayoritario y minoritario. Estos resultados vienen a indicar que la enseñanza colaborativa es una práctica mejor percibida por los estudiantes del grupo mayoritario de EF, Arte e Idiomas que del minoritario del mismo ámbito de estudio.

Se analizaron las diferencias en los contenidos formativos abordados en la carrera sobre atención a la diversidad e inclusión en función del ámbito de estudio y grupo étnico. La Tabla 4 contiene los resultados de este factor. Puede observarse que ni el efecto principal de la variable ámbito de estudio $[F(2,469)=.2 .58, p=0.77]$, ni el efecto principal del grupo étnico $[F(1,469)=.236, p=.627]$ resultaron estadísticamente significativos. Sin embargo, en el ítem 2 'Implementación de adaptaciones instructivas' y en el 6 'Procesos de reflexión crítica sobre estereotipos y creencias acerca de la diversidad' se apreciaron diferencias estadísticamente significativas atribuibles al ámbito de estudio. Las pruebas post hoc mostraron que, en el primer caso, los estudiantes de Ciencias Sociales y Educación ( $M=$ $2.55 ; D T=.85)$ y los de Ciencia y Tecnología $(M=2.53 ; D T=.92)$ indicaban haber abordado contenidos acerca de la implementación de adaptaciones instructivas como 
medida de respuesta a las diferencias por capacidad en grado significativamente mayor que los de Educación Física, Arte e Idiomas $(M=2.46 ; D T=.88)$. En el segundo caso, los resultados mostraban que los estudiantes de Ciencias Sociales y Educación $(M=2.31$; $D T=$ .92) habían analizado y debatido las creencias y estereotipos sobre la diversidad humana en grado significativamente mayor que los de Educación Física, Arte e Idiomas $(M=1.96 ; D T$ $=.84)$ y los de Ciencia y Tecnología $(M=1.91 ; D T=.83)$.

Tabla 4. Contenidos formativos abordados en la carrera en función del ámbito de estudio y grupo étnico (Contraste de medias)

\begin{tabular}{|c|c|c|c|c|c|c|c|c|c|c|}
\hline & \multicolumn{2}{|c|}{$\begin{array}{c}\text { CC.SS. y } \\
\text { Educ }\end{array}$} & \multicolumn{2}{|c|}{$\begin{array}{l}\text { EF, Arte, } \\
\text { Idiomas }\end{array}$} & \multicolumn{2}{|c|}{ CC TE } & \multirow[b]{2}{*}{ Efect } & \multirow[b]{2}{*}{$\boldsymbol{F}$} & \multirow[b]{2}{*}{$\boldsymbol{G l}$} & \multirow[b]{2}{*}{$p$} \\
\hline & $M$ & $D T$ & $M$ & $D T$ & $M$ & $D T$ & & & & \\
\hline Total factor & & & & & & & & & & \\
\hline Grupo mayoritario & 15.0 & 3.79 & 14.4 & 3.75 & 14.2 & 3.50 & A & 2.58 & 2 & .077 \\
\hline Grupo minoritario & 14.6 & 3.73 & 12.8 & 3.70 & 15.5 & 4.34 & G & .24 & 1 & 627 \\
\hline $\begin{array}{l}\text { 1. Diseño de } \\
\text { adaptaciones } \\
\text { curriculares }\end{array}$ & & & & & & & $A x G$ & 1.96 & 2 & .142 \\
\hline - $\quad$ Mayoritario & 2.27 & .91 & 2.10 & .86 & 2.02 & .82 & A & 1.68 & 2 & 187 \\
\hline - $\quad$ Minoritario & 2.16 & .84 & 1.95 & .94 & 2.64 & 1.12 & $\begin{array}{c}\mathrm{G} \\
\mathrm{AxG}\end{array}$ & $\begin{array}{l}.881 \\
2.96\end{array}$ & $\begin{array}{l}1 \\
2\end{array}$ & $\begin{array}{l}.347 \\
.052\end{array}$ \\
\hline
\end{tabular}

2. Implementación de adaptaciones instructivas

- Mayoritario

- Minoritario

$\begin{array}{llllllllll}\mathbf{2 . 5 5} & .85 & \mathbf{2 . 4 6} & .88 & \mathbf{2 . 5 3} & .92 & \mathrm{~A} & 3.21 & 2 & .041^{*} \\ \mathbf{2 . 3 4} & .86 & \mathbf{2 . 1 0} & .95 & \mathbf{2 . 9 1} & .94 & \mathrm{G} & .248 & 1 & .619 \\ & & & & & & \text { AxG } & 2.42 & 2 & .090\end{array}$

3. Estrategias organizativas (agrupamiento)

- Mayoritario

- Minoritario

$\begin{array}{ll}2.61 & .89\end{array}$

$\mathbf{2 . 6 3} \quad .88$

$\begin{array}{ll}2.59 & .87\end{array}$

A 2.68

0.70

4. Estrategias de

$2.56-91$

$2.10 \quad .88$

$2.91 \quad .83$

$\mathrm{G} \quad .474 \quad 1 \quad .492$

enseñanza colaborativa

- Mayoritario

- Minoritario

$\begin{array}{llll}\mathbf{2 . 5 9} & .83 & \mathbf{2 . 5 6} & .95\end{array}$

$\begin{array}{ll}2.55 & .77\end{array}$

$2.38 \quad .92$

$\mathbf{2 . 1 8} .87$

$\begin{array}{cccc}\mathrm{A} & .942 & 2 & .391 \\ \mathrm{G} & 2.32 & 1 & .128 \\ \mathrm{AxG} & .641 & 2 & .466\end{array}$

5. Estrategias y recursos de evaluación auténtica

- Mayoritario

$\begin{array}{llll}\mathbf{2 . 7 2} & .87 & \mathbf{2 . 7 4} & .90\end{array}$

$\begin{array}{ll}2.68 & .86\end{array}$ $2.38 \quad .86$

$2.73 \quad .78$

$\begin{array}{cccc}\mathrm{A} & .453 & 2 & .355 \\ \mathrm{G} & 1.08 & 1 & .849 \\ \mathrm{AxG} & .791 & 2 & .620\end{array}$

6. Procesos de reflexión crítica 


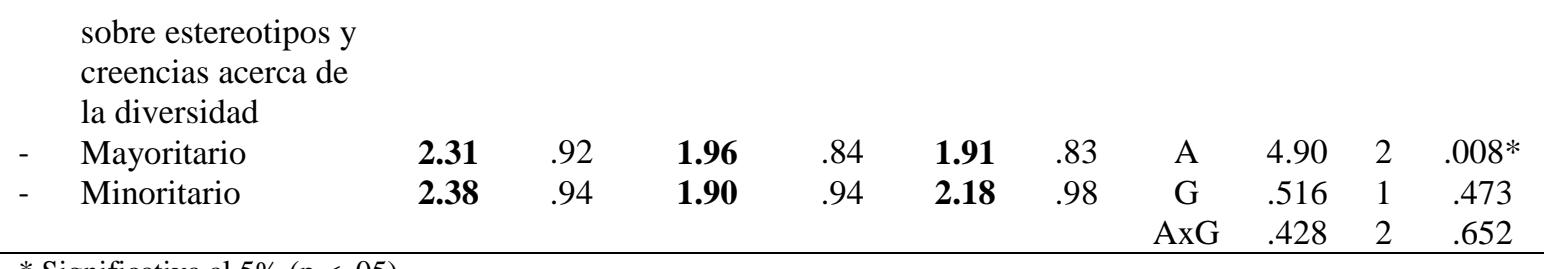

* Significativa al $5 \%(\mathrm{p}<.05)$

$1=$ Nada $; 2=$ Algo $; 3=$ Bastante $; 4=$ Mucho

El efecto de la interacción A x G tampoco resultó significativo $[F(2,469)=1.96, p=.142]$ ni sobre el factor en su conjunto ni sobre los ítems por separado, exceptuado el ítem 3 'Estrategias instructivas (agrupamiento)'. Mientras la capacitación recibida en el uso de estas estrategias por el grupo mayoritario de los tres ámbitos de estudio fue similar, no ocurrió así dentro del grupo minoritario en el que los participantes de Ciencia y Tecnología $(M=2.91 ; D T=.83)$ valoraron la formación recibida significativamente mejor que los de Ciencias Sociales y de la Educación $(M=2.56 ; D T=.91)$ y de Educación Física, Arte e idiomas $(M=2.10 ; D T=.88)$. Estos resultados indican que el abordaje de estos contenidos no fue percibido por igual dentro del grupo mayoritario que minoritario en el seno del cual los estudiantes de Ciencia y Tecnología eran los más satisfechos.

\section{CONCLUSIONES}

Los estudiantes de la UPNFM tienen percepciones favorables hacia los principios y planteamientos de la atención a la diversidad y la inclusión. Sin embargo, durante la formación en la carrera el grado de abordaje de contenidos como las adaptaciones instructivas, la adaptación curricular, las estrategias organizativas y el estudio de estereotipos sobre la diversidad ha sido bajo. Por otro lado, los estudiantes perciben que las competencias necesarias para el desempeño docente en atención a la diversidad consisten en la capacidad de reflexionar sobre la diversidad, la capacidad para proponer y desarrollar estrategias de atención en diferentes contextos y, finalmente, la capacidad para implementar adaptaciones instructivas en el aula.

La comparación de las percepciones de los estudiantes según ámbito de estudio y grupo étnico demostró que no existen diferencias estadísticamente significativas. Es decir, que los 
participantes, independientemente del ámbito de sus estudios o de su pertenencia a un grupo étnico mayoritario o minoritario, tenían percepciones básicamente similares de la atención a la diversidad y la inclusión, salvo alguna excepción previamente comentada.

\section{RECOMENDACIONES}

Consideramos que futuras investigaciones deberían estar orientadas a estudiar con mayor grado de profundidad la formación inicial de los estudiantes que pertenecen a grupos étnicos $\mathrm{u}$ otros colectivos minoritarios que tienen presencia en las instituciones universitarias. Pues éstos maestros pueden jugar un papel fundamental en la atención a la diversidad y la inclusión por el simple hecho de constituir minorías.

Otro aspecto importante sería incluir en esta clase de estudios, al profesorado universitario. Indagar sobre sus creencias, actitudes y percepciones acerca de la diversidad humana y la inclusión es un objeto de estudio que guarda especial interés para la formación inicial. 


\section{REFERENCIAS BIBLIOGRÁFICAS}

Aguilar, L. (2008). Documento base reforma curricular Universidad Pedagógica Nacional Francisco Morazán. Tegucigalpa: Sello Editorial UPNFM.

Arranz, P. (2003). La formación inicial del profesorado: Modelos, demandas y prescripciones. Anuario De Pedagogía, 5(1), 75-101.

Campos, M. (2003). El rol de las universidades pedagógicas en la formación de docentes: Experiencias y desafios. Chile: UNESCO.

Cardona, M. C. (2002). Introducción a los métodos de investigación en educación. Madrid: Editorial EOS

Durán, D., y Giné, C. (2011). La formación del profesorado para la educación inclusiva: Un proceso de desarrollo profesional y de mejora de los centros para atender la diversidad. Revista Latinoamericana de Educación inclusiva, 5(2), 153-170

León, M. J. (1998). De la integración escolar a la escuela inclusiva o escuela para todos. En Royo, L., y López, N. (Eds.), Bases psicopedagógicas de la educación especial (pp. 39-59) Madrid:Pirámide.

Mason, T. C. (1997). Urban field experiences and prospective teachers' attitudes toward inner-city schools. Teacher Education Quarterly, 29-40

Sales, A. (2006). La formación inicial del profesorado ante la diversidad: Una propuesta metodológica para el nuevo espacio europeo de educación superior. Revista Interuniversitaria de Formación de Profesorado, 20(3), 201-217.

Torres, J. A. (2009). Dilemas y necesidades de formación del profesorado en el marco de una escuela comprensiva que atiende a la diversidad. Salamanca: Ediciones Universidad de Salamanca.

UNESCO. (1990). Declaración mundial sobre educación para todos: satisfacción de las necesidades básicas de aprendizaje. Jomtiem, Tailandia: Autor

UNESCO. (2000). Educación para todos: cumplir nuestros compromisos comunes, aprobado por el foro mundial sobre la educación. Dakar, Senegal: Autor

UNESCO. (2012). Antecedentes y criterios para la elaboración de políticas docentes en América latina y el Caribe. Santiago de Chile: UNESCO.

UPNFM. (2002). Documento base del Proyecto Atención a la Diversidad. Tegucigalpa: UPNFM. 
